\title{
Article
}

\section{The Use of Cabozantinib in Advanced Hepatocellular Carcinoma in Hong Kong-A Territory-Wide Cohort Study}

\author{
Jeffrey Sum-Lung Wong ${ }^{1,+}{ }^{+}$, Yawen Dong ${ }^{1,+}$, Vikki Tang ${ }^{1}$, Thomas Leung ${ }^{2}$, Cynthia S. Y. Yeung ${ }^{3}$, Anna Tai ${ }^{4}$, \\ Ada Law ${ }^{5}$, Tracy Shum ${ }^{6}$, Gerry Gin-Wai Kwok ${ }^{1}$, Bryan Cho-Wing Li ${ }^{1}$, Roland Leung ${ }^{1}$, Joanne Chiu ${ }^{1}$, \\ Ka-Wing Ma ${ }^{7}$, Wong-Hoi She ${ }^{7}$, Josephine Tsang ${ }^{1}$, Tan-To Cheung ${ }^{7}$ and Thomas Yau ${ }^{1, * \mathbb{C}}$
}

1 Department of Medicine, Queen Mary Hospital, The University of Hong Kong, Hong Kong, China; wsl714@ha.org.hk (J.S.-L.W.); yawen.dong@gesundheitsverbund.at (Y.D.); vyftang@hku.hk (V.T.); kgw951@ha.org.hk (G.G.-W.K.); lcw027@ha.org.hk (B.C.-W.L.); lcy035@ha.org.hk (R.L.); jwychiu@hku.hk (J.C.); twy917@ha.org.hk (J.T.)

2 Department of Medicine, Hong Kong Sanatorium and Hospital, Hong Kong, China; thomas.wt.leung@hksh.com

3 Department of Clinical Oncology, Tuen Mun Hospital, Hong Kong, China; Syyeung@ha.org.hk

4 Department of Clinical Oncology, Queen Elizabeth Hospital, Hong Kong, China; typ065@ha.org.hk

5 Department of Clinical Oncology, Pamela Youde Nethersole Eastern Hospital, Hong Kong, China; lawly@ha.org.hk

6 Department of Clinical Oncology, Princess Margaret Hospital, Hong Kong, China; scy409@ha.org.hk

7 Department of Surgery, Queen Mary Hospital, The University of Hong Kong, Hong Kong, China; kawingma@hku.hk (K.W.-M.); swh180@ha.org.hk (W.H.-S.); cheung68@hku.hk (T.T.-C.)

* Correspondence: tyaucc@hku.hk; Tel.: +852-2255-3111

+ Co-First Author

check for

updates

Citation: Wong, J.S.-L.; Dong, Y.; Tang, V.; Leung, T.; Yeung, C.S.Y.; Tai, A.; Law, A.; Shum, T.; Kwok, G.G.-W.; Li, B.C.-W.; et al. The Use of Cabozantinib in Advanced Hepatocellular Carcinoma in Hong Kong-A Territory-Wide Cohort Study. Cancers 2021, 13, 2002. https:// doi.org/10.3390/cancers13092002

Academic Editor: Lorenza Rimassa

Received: 23 March 2021

Accepted: 17 April 2021

Published: 21 April 2021

Publisher's Note: MDPI stays neutral with regard to jurisdictional claims in published maps and institutional affiliations.

Copyright: (c) 2021 by the authors. Licensee MDPI, Basel, Switzerland. This article is an open access article distributed under the terms and conditions of the Creative Commons Attribution (CC BY) license (https:// creativecommons.org/licenses/by/ $4.0 /)$.
Simple Summary: The vascular endothelial growth factor and c-MET pathways are strongly implicated in hepatocellular carcinoma (HCC). Cabozantinib inhibits both pathways and has been approved in sorafenib-exposed advanced HCC (aHCC). We aimed to evaluate the real-life pattern of use, efficacy, and safety of cabozantinib in aHCC patients in a territory-wide study. In single-agent cabozantinib patients $(n=27)$, we found that $3.7 \%$ had a response, $44.4 \%$ had disease control, and the median overall survival (OS) was 8.28 months. Around $74.1 \%$ of patients had adverse events (AEs). We also did an exploratory analysis of patients who received cabozantinib as an add-on to immunecheckpoint inhibitors (ICIs) after progression on ICIs. Out of 15 such patients, $6.7 \%$ had a response and the median OS was 15.1 months, with $73.3 \%$ of patients having AEs. Overall, cabozantinib had good efficacy, survival, and safety in aHCC patients in a real-life setting.

Abstract: (1) Background: Cabozantinib is approved in sorafenib-exposed advanced hepatocellular carcinoma (aHCC). We evaluated the real-life pattern of use, efficacy, and tolerability of cabozantinib in aHCC. (2) Methods: This territory-wide study included consecutive aHCC patients who received cabozantinib between February 2018 and September 2020 in Hong Kong. The objective response rate (ORR), disease control rate (DCR), overall survival (OS), and adverse events (AE) were assessed. (3) Results: Overall, 42 patients were included. Approximately 83.3\% had Child-Pugh A cirrhosis. About $64.3 \%$ received cabozantinib as a single agent, and the remaining $35.7 \%$ received cabozantinib as an add-on to immune checkpoint inhibitors (ICIs). For single-agent patients, the median follow-up was 6.7 months. The ORR was 3.7\%, DCR was $44.4 \%$, and the median OS was 8.28 months. About $74.1 \%$ of patients experienced any AEs with $7.4 \%$ having grade $\geq 3$ AEs. Among patients who received prior ICIs $(n=16)$, the ORR was $6.3 \%$, and the median OS was 8.28 months. An exploratory analysis of patients who received cabozantinib as an add-on to ICIs showed an ORR of $6.7 \%$ and a median OS of 15.1 months, with $73.3 \%$ having any $\mathrm{AE}$ and $13.3 \%$ having grade $\geq 3$ AEs. (4) Conclusions: Cabozantinib had good anti-tumor activity, survival benefits, and acceptable tolerability in real-life aHCC patients.

Keywords: hepatocellular carcinoma; cabozantinib; tyrosine kinase inhibitors 


\section{Introduction}

The majority of hepatocellular carcinomas (HCCs) present as advanced disease not amenable to surgery [1]. Vascular endothelial growth factor (VEGF)-mediated cellular signaling pathways have been implicated in the pathogenesis of HCC and studied extensively as a therapeutic target [2]. Sorafenib, a small molecule tyrosine kinase inhibitor (TKI) that inhibits VEGF pathways, was the first agent licensed for use in the systemic treatment of HCC. In the landmark SHARP trial, sorafenib demonstrated a superior median overall survival (OS) (10.7 months vs. 7.9 months, $p<0.001)$ and median time to progression (5.5 months vs. 2.8 months, $p<0.001$ ) compared to the placebo [3]. Lenvatinib demonstrated non-inferiority to sorafenib (median OS 13.6 months vs. 12.3 months) in the REFLECT trial and was duly licensed for use as first-line treatment of advanced HCC (aHCC) [4]. Regorafenib became the first ever agent to be licensed for use in second-line treatment for patients who progressed on sorafenib after demonstrating significant improvement in OS (hazard ratio $0.63, p<0.0001$, median OS 10.6 months vs. 7.8 months) compared to the placebo in this population in the RESORCE trial [5]. Finally, ramucirumab, a VEGFR-2 inhibitor, was licensed for use in sorafenib-treated aHCC with alpha-fetoprotein (AFP) $\geq 400 \mathrm{ng} / \mathrm{mL}$ after demonstrating superior OS (median OS 8.5 vs. 7.3 months, $p=0.0199$ ) compared to the placebo in the REACH-2 trial [6].

The c-Met and AXL receptor tyrosine kinases promote epithelial-to-mesenchymal transition, invasion, and metastasis in human malignancies [7-9]. Additionally, the c-Met pathway has been found to be up-regulated in HCCs treated with sorafenib, implicating it in sorafenib resistance $[10,11]$. The multi-kinase inhibitor cabozantinib has activity against VEGF receptors 1-3, c-Met, and the TAM receptors (Tyro-3, AXL, and Mer), thus conferring it the theoretical benefit of overcoming sorafenib resistance in HCC $[10,12,13]$. In the CELESTIAL trial, cabozantinib demonstrated significantly superior overall survival (median OS 10.2 months vs. 8.0 months, $p=0.005$ ), PFS (5.2 months vs. 1.9 months, $p<0.001)$, and objective response rate ( $4 \%$ vs. $\leq 1 \%, p=0.009)$ compared to the placebo in patients with sorafenib-treated HCC [14]. This led to cabozantinib's approval as a treatment for patients with sorafenib-treated HCC in second- or third-line settings.

Despite the encouraging results of the CELESTIAL trial, important questions regarding the use of cabozantinib in aHCC remain. Firstly, there may be significant differences in the outcome and safety of cabozantinib in real-life use compared to trial settings. These potential differences may be due to trial exclusion criteria such as clinical and laboratory limits on liver and hematological function [14] as well as the time needed for trial screening, both of which likely precluding a significant number of patients with advanced cirrhosis or aggressive HCC from trial participation. Secondly, since the official launch of cabozantinib worldwide, options of systemic HCC treatment have undergone a significant expansion. In particular, multiple immune checkpoint inhibitors (ICIs) have been approved both in firstand second-line settings. These include atezolizumab-bevacizumab in the first line based on the phase III IMbrave150 trial [15,16], nivolumab [17], nivolumab-ipilimumab [18], and pembrolizumab [19] as second-line treatment based on the single-arm phase I/II CheckMate-040 and Keynote-224 trials. However, it should be noted that nivolumab and pembrolizumab monotherapy did not reach pre-defined endpoints in the subsequent phase III studies [20,21]. Despite there being a small subgroup of CELESTIAL patients having received ICIs [22], the efficacy of cabozantinib after ICIs is largely unknown. We thus conducted a territory-wide study of aHCC patients who received cabozantinib in a real-life setting.

\section{Methods}

This was a multi-center, territory-wide retrospective analysis involving six tertiary /quaternary oncology centers in Hong Kong. The study was approved by the University of Hong Kong/Hospital Authority Hong Kong West Cluster Institutional Review Board and was conducted based on the principles of the Declaration of Helsinki. Between February 2018 and September 2020, consecutive patients with HCC not amenable to loco- 
regional therapy who received cabozantinib were included. Data were retrieved from the anonymized territory-wide electronic records of the Hong Kong Hospital Authority (HA), which covers $90 \%$ of all secondary and tertiary care in the territory.

HCCs were diagnosed either according to the European Association for the Study of the Liver (EASL) combined criteria when both an elevated serum AFP and characteristic radiological findings were present, or by histological confirmation with operative specimens, fine needle aspiration, or biopsy. Staging was done by computer tomography or magnetic resonance imaging according to the Barcelona Clinic Liver Cancer (BCLC) system. Relevant clinical, imaging, and laboratory information were gathered from the aforementioned source.

\subsection{Dosing, Evaluation, and Follow-Up}

The starting doses of cabozantinib in individual patients were decided by treating physicians. Tumor responses were evaluated according to both the Response Evaluation Criteria in Solid Tumor version 1.1 (RECIST 1.1) and the modified RECIST (mRECIST) for HCC $[23,24]$. The best objective response (BOR) was defined as the best response compared to baseline per RECIST 1.1 or mRECIST. The objective response rate (ORR) was defined as the percentage of patients with a complete response (CR) or a partial response (PR). The disease control rate (DCR) was defined as the percentage of patients with CR, PR, or stable disease (SD). Reassessment scans were generally scheduled every 8-12 weeks. For responses to prior ICIs, primary resistance was defined as always having progressive disease, while acquired resistance was defined as ever having stable or responding disease. Adverse events (AEs) were graded with the National Cancer Institute's Common Terminology Criteria for Adverse Events (NCI CTCAE) version 4.0 [25]. Patients were followed up with regular physical examination, blood tests, and scanning.

\subsection{Statistical Analysis}

Statistical analyses were performed using SPSS (version 26, IBM, Armonk, NY, USA). Follow-up time was calculated from the date of the first dose to death or last follow-up. Overall survival (OS) was calculated from the date of the first dose to death from any cause or censored at the last follow-up if the patient was still alive. Time to progression (TTP) was calculated from the date of the first dose to tumor progression or censored at the last follow-up or death while on treatment for patients without progression. The median OS, TTP, and survival rates were estimated by Kaplan-Meier analyses, and survival curves were compared using log-rank tests. The median follow-up time was estimated using the reverse Kaplan-Meier method. Categorical variables were compared using Pearson's chi-squared test or Fisher's exact test. Nonparametric statistics were used to compare continuous variables. A $p$-value of 0.05 was taken as cut-off for statistical significance.

\section{Results}

\subsection{Demographics}

Forty-two patients were included in the study. Table 1 and Table S1 show their baseline characteristics. The median age was 59.5 (range 41-85). Most patients had Child-Pugh (CP) grade A cirrhosis (83.3\%), and 31\% were of Albumin-Bilirubin (ALBI) grade 1. About $88.1 \%$ had hepatitis B-related HCC. Notably, $64.3 \%$ of analyzed patients received single-agent cabozantinib $(n=27)$, while the remaining $35.7 \%$ patients used cabozantinib as add-on therapy to ongoing ICIs (anti-PD1 with or without anti-CTLA-4) (Table S2). 
Table 1. Baseline characteristics.

\begin{tabular}{|c|c|c|c|c|}
\hline & & $\begin{array}{c}\text { All Patients } \\
(n=42)\end{array}$ & $\begin{array}{l}\text { Single Agent } \\
(n=27)\end{array}$ & $\begin{array}{c}\text { Combination } \\
(n=15)\end{array}$ \\
\hline $\begin{array}{l}\text { Median age } \\
\text { (range), years }\end{array}$ & & $59.5(41-85)$ & $58(41-85)$ & $64(42-79)$ \\
\hline Male, $n(\%)$ & & $36(85.7 \%)$ & $25(92.6 \%)$ & $11(73.3 \%)$ \\
\hline \multirow[t]{4}{*}{$\begin{array}{l}\text { Hepatocellular } \\
\text { carcinoma } \\
\text { etiology, } n(\%)\end{array}$} & Hepatitis B (HBV) & $37(88.1 \%)$ & $23(85.2 \%)$ & $14(93.3 \%)$ \\
\hline & Hepatitis C (HCV) & $2(4.8 \%)$ & $1(3.7 \%)$ & $1(6.7 \%)$ \\
\hline & Alcoholic & $1(2.4 \%)$ & $1(3.7 \%)$ & 0 \\
\hline & $\begin{array}{c}\text { Non-alcoholic } \\
\text { Steatohepatitis } \\
\text { (NASH) }\end{array}$ & $2(4.8 \%)$ & $2(7.4 \%)$ & 0 \\
\hline \multirow[t]{3}{*}{ Child-Pugh, $n(\%)$} & $\mathrm{A}$ & $35(83.3 \%)$ & $23(85.2 \%)$ & $12(80 \%)$ \\
\hline & $\mathrm{B}$ & $6(14.3 \%)$ & $4(14.8)$ & $2(13.3 \%)$ \\
\hline & $\mathrm{C}$ & $1(2.4 \%)$ & 0 & $1(6.7 \%)$ \\
\hline \multirow[t]{3}{*}{$\begin{array}{c}\text { Albumin- } \\
\text { Bilirubin Grade, } \\
n(\%)\end{array}$} & 1 & $13(31 \%)$ & $7(25.9 \%)$ & $6(40 \%)$ \\
\hline & 2 & $24(57.1 \%)$ & $16(59.3 \%)$ & $8(53.3 \%)$ \\
\hline & 3 & $5(11.9 \%)$ & $4(14.8 \%)$ & $1(6.7 \%)$ \\
\hline \multirow[t]{3}{*}{$\begin{array}{c}\text { Barcelona Clinic } \\
\text { Liver Cancer stage, } \\
n(\%)\end{array}$} & B & $2(4.8 \%)$ & $1(3.7 \%)$ & $1(6.7 \%)$ \\
\hline & $\mathrm{C}$ & $39(92.9 \%)$ & $26(96.3 \%)$ & $13(86.7 \%)$ \\
\hline & $\mathrm{D}$ & $1(2.4 \%)$ & 0 & $1(6.7 \%)$ \\
\hline \multirow[t]{2}{*}{$\begin{array}{c}\text { Baseline } \\
\text { Performance } \\
\text { Status, } n(\%)\end{array}$} & 01 & $40(95.2 \%)$ & $27(100 \%)$ & $13(86.7 \%)$ \\
\hline & 2 & $2(4.8 \%)$ & 0 & $2(13.3 \%)$ \\
\hline $\begin{array}{l}\text { Alpha-fetoprotein } \\
\geq 400 \mathrm{ng} / \mathrm{mL}, n \\
(\%)\end{array}$ & & $21(50 \%)$ & $13(48.1 \%)$ & $8(53.3 \%)$ \\
\hline $\begin{array}{c}\text { Extrahepatic } \\
\text { metastases, } n(\%)\end{array}$ & & $37(88.1 \%)$ & $24(88.9 \%)$ & $13(86.7 \%)$ \\
\hline $\begin{array}{c}\text { Vascular invasion, } \\
n(\%)\end{array}$ & & $7(16.7 \%)$ & $6(22.2 \%)$ & $1(6.7 \%)$ \\
\hline \multirow[t]{4}{*}{$\begin{array}{l}\text { Cabozantinib } \\
\text { setting, } n(\%)\end{array}$} & Second line & $15(35.7 \%)$ & $13(48.1 \%)$ & $2(13.3 \%)$ \\
\hline & Third line & $9(21.4 \%)$ & $6(22.2 \%)$ & $3(20 \%)$ \\
\hline & Fourth line & $7(16.7 \%)$ & $3(11.1 \%)$ & $4(26.7 \%)$ \\
\hline & Fifth line and beyond & $11(26.2 \%)$ & $5(18.5 \%)$ & $6(40 \%)$ \\
\hline \multirow[t]{5}{*}{$\begin{array}{c}\text { Previous therapies, } \\
n(\%)\end{array}$} & Curative resection & $21(50 \%)$ & $9(33.3 \%)$ & $12(80 \%)$ \\
\hline & Radiotherapy & $10(23.8 \%)$ & $6(22.2 \%)$ & $4(26.7 \%)$ \\
\hline & $\begin{array}{c}\text { Transarterial } \\
\text { chemoembolization }\end{array}$ & $20(47.6 \%)$ & $13(48.1 \%)$ & 7 (46.7\%) \\
\hline & $\begin{array}{c}\text { Any tyrosine kinase } \\
\text { inhibitors }\end{array}$ & $40(95.2 \%)$ & $26(96.3 \%)$ & $14(93.3 \%)$ \\
\hline & $\begin{array}{c}\text { Any immune } \\
\text { checkpoint inhibitors }\end{array}$ & $31(73.8 \%)$ & $16(59.3 \%)$ & $15(100 \%)$ \\
\hline
\end{tabular}

Regarding prior treatments, the patients in our cohort were generally heavily pretreated. Around $35.7 \%$ of patients received cabozantinib as second-line therapy, and $21.4 \%$ of patients received cabozantinib as third-line therapy. The remaining $16.7 \%$ and $26.2 \%$ of 
patients received cabozantinib as their 4 th and $\geq 5$ th line of systemic treatment, respectively. Of note, single-agent cabozantinib was most often used as second-line therapy, while cabozantinib-ICI combinations were more frequently used as salvage therapy in fourth line or beyond. The vast majority of patients were exposed to tyrosine kinase inhibitors $(95.2 \%)$, with $66.7 \%$ exposed to lenvatinib, and $40.5 \%$ to sorafenib. Notably, 31 (73.8\%) patients had prior exposure to ICIs before cabozantinib. The majority of patients started cabozantinib after progression on prior regimes, with some due to side effects.

\subsection{Outcomes of Single-Agent Cabozantinib}

\subsubsection{Clinical Outcomes}

The median follow-up was 6.74 months (95\% C.I., 3.35-10.1). At the time of study-cut off, six patients were lost to follow-up. Table 2 summarizes the BORs. The ORR and DCR were $3.7 \%$ and $44.4 \%$, respectively. There were no differences in the BORs by the RECIST 1.1 and mRECIST criteria. The median TTP was 3.88 months (95\% C.I., 2.31-5.45). The median OS was 8.28 months (95\% C.I., 3.91-12.7) (Figure 1). The 6-month and 1-year survival rates were $61.1 \%$ and $17.8 \%$, respectively.

Table 2. Best objective response, all patients.

\begin{tabular}{cccc}
\hline Activity, $\boldsymbol{n}(\mathbf{\%})$ & All Patients $(\boldsymbol{n = 4 2 )}$ & Single Agent $(\boldsymbol{n = 2 7 )}$ & Combination $(\boldsymbol{n}=\mathbf{1 5})$ \\
\hline Progressive disease & $18(42.9 \%)$ & $10(37.0 \%)$ & $8(53.3 \%)$ \\
\hline Stable disease & $14(33.3 \%)$ & $11(40.7 \%)$ & $3(20 \%)$ \\
\hline Partial response & $2(4.8 \%)$ & $1(3.7 \%)$ & $1(6.7 \%)$ \\
\hline Complete response & $0(0 \%)$ & 0 & $3(20 \%)$ \\
\hline Non-evaluable & $8(19.0 \%)$ & $5(18.5 \%)$ & $6.7 \%$ \\
\hline $\begin{array}{c}\text { Objective response } \\
\text { rate }\end{array}$ & $4.8 \%$ & $3.7 \%$ & $26.7 \%$ \\
\hline Disease control rate & $38.1 \%$ & $44.4 \%$ &
\end{tabular}

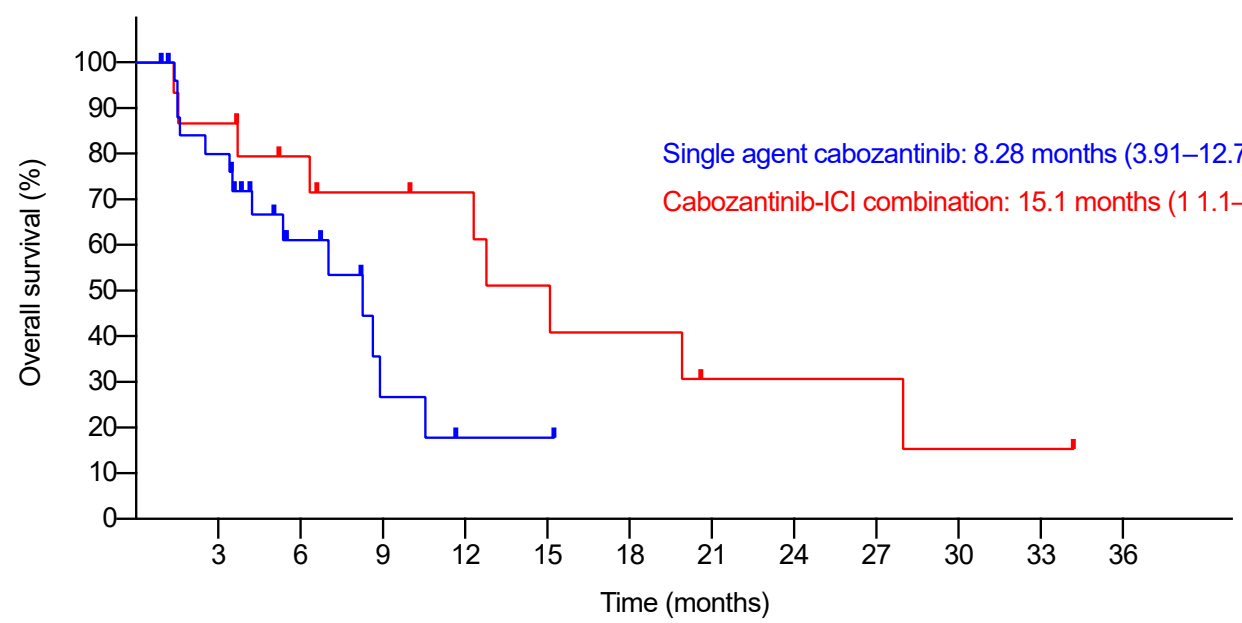

Figure 1. Kaplan-Meier analysis of OS of single-agent and cabozantinib-ICI combination patients.

\subsubsection{Dosing and Adverse Events}

The median treatment duration was 2.27 months (range 0.2-11.7). The median daily dose was $40 \mathrm{mg}$ (range 20-60). At the study cut-off, $44.4 \%$ of patients terminated treatment due to progressive disease, $14.8 \%$ due to intolerance, $3.7 \%$ due to non-cancer death, and $22.2 \%$ due to unrelated medical conditions and other causes. Around $14.8 \%$ of patients were still receiving treatment. About $25.9 \%$ of patients received other subsequent systemic treatments. 
Table 3 shows all AEs that were observed in the study. The most common AEs were hand-foot syndrome (HFS), hypertension, malaise, diarrhea, and transaminitis. A total of $74.1 \%$ and $7.4 \%$ of patients had all grade and grade $\geq 3 \mathrm{AEs}$, respectively. One patient had grade 3 hypertension and another had grade 3 HFS. Overall, 22.2\% of patients required dose interruptions, $25.9 \%$ required dose reduction, and $14.8 \%$ required treatment termination due to AEs. There were no treatment-related deaths.

Table 3. Adverse events (AEs).

\begin{tabular}{|c|c|c|c|c|}
\hline & & $\begin{array}{l}\text { All Patients } \\
\quad(n=42)\end{array}$ & $\begin{array}{l}\text { Single Agent } \\
\quad(n=27)\end{array}$ & $\begin{array}{l}\text { Combination } \\
(n=15)\end{array}$ \\
\hline All AE & & $31(73.8 \%)$ & $20(74.1 \%)$ & $11(73.3 \%)$ \\
\hline$\geq$ Grade $3 \mathrm{AE}$ & & $4(9.5 \%)$ & $2(7.4 \%)$ & $2(13.3 \%)$ \\
\hline $\begin{array}{l}\text { AE requiring dose } \\
\text { interruption }\end{array}$ & & $8(19.0 \%)$ & $6(22.2 \%)$ & $2(13.3 \%)$ \\
\hline $\begin{array}{l}\text { AE requiring dose } \\
\text { reduction }\end{array}$ & & $10(23.8 \%)$ & $7(25.9 \%)$ & $3(20 \%)$ \\
\hline $\begin{array}{l}\text { AE requiring } \\
\text { treatment cessation }\end{array}$ & & $6(14.3 \%)$ & $4(14.8 \%)$ & $2(13.3 \%)$ \\
\hline \multicolumn{5}{|l|}{ Hand-Foot Syndrome } \\
\hline & Grade 1-2 & $12(28.6 \%)$ & $8(29.6 \%)$ & $4(26.7 \%)$ \\
\hline & Grade 3 & $2(4.8 \%)$ & $1(3.7 \%)$ & $1(6.7 \%)$ \\
\hline \multicolumn{5}{|l|}{ Hypertension } \\
\hline & Grade 1-2 & $6(14.3 \%)$ & $5(18.5 \%)$ & $1(6.7 \%)$ \\
\hline & Grade 3 & $2(4.8 \%)$ & $1(3.7 \%)$ & $1(6.7 \%)$ \\
\hline Transaminitis & & $6(14.3 \%)$ & $4(14.8 \%)$ & $2(13.3 \%)$ \\
\hline Diarrhea & & $6(14.3 \%)$ & $5(18.5 \%)$ & $1(6.7 \%)$ \\
\hline Nausea/vomiting & & $3(7.1 \%)$ & $2(7.4 \%)$ & $1(6.7 \%)$ \\
\hline $\begin{array}{c}\text { Malaise/Loss of } \\
\text { appetite }\end{array}$ & & $7(16.7 \%)$ & $6(22.2 \%)$ & $1(6.7 \%)$ \\
\hline $\begin{array}{c}\text { Rash/Other Skin } \\
\text { issues }\end{array}$ & & $6(14.3 \%)$ & $4(14.8 \%)$ & $2(13.3 \%)$ \\
\hline Musculoskeletal pain & & $2(4.8 \%)$ & $1(3.7 \%)$ & $1(6.7 \%)$ \\
\hline Proteinuria & & $5(11.9 \%)$ & $4(14.8 \%)$ & $1(6.7 \%)$ \\
\hline Neutropenia & & $4(9.5 \%)$ & $4(14.8 \%)$ & 0 \\
\hline Thrombocytopenia & & $3(7.1 \%)$ & $3(11.1 \%)$ & 0 \\
\hline Epigastric Discomfort & & $1(2.4 \%)$ & $1(3.7 \%)$ & 0 \\
\hline Palpitations & & $1(2.4 \%)$ & $1(3.7 \%)$ & 0 \\
\hline Hypothyroidism & & $2(4.8 \%)$ & $1(3.7 \%)$ & $1(6.7 \%)$ \\
\hline
\end{tabular}

\subsubsection{Single-Agent Cabozantinib Post-Immune Checkpoint Inhibitors}

As mentioned above, 16 (59.3\%) patients had prior exposure to ICIs before receiving single-agent cabozantinib. The median interval between the last dose of previous ICI and cabozantinib was 5.36 weeks (range 1.71-84). Three patients discontinued prior ICIs due to AEs, with the remaining discontinuations due to progressive disease. Approximately $37.5 \%$ of patients had acquired resistance, and $43.8 \%$ had primary resistance to prior ICIcontaining regimes. Overall, the ORR was $6.3 \%$, the DCR was $31.3 \%$, the median TTP was 3.51 months (95\% C.I., 1.22-5.82), and the median OS was 8.28 months (95\% C.I., 1.33-15.2) (Table 4). There was no significant difference in the median OS among patients with primary resistance to prior ICI regimes compared to those with acquired resistance 
(primary resistance 3.52 months (95\% C.I., 0-10.0), acquired resistance 7.03 months (95\% C.I., $0-15.9), p=0.891)$.

Table 4. Best objective response, post-immune checkpoint inhibitors patients.

\begin{tabular}{cccc}
\hline Activity, $\boldsymbol{n}(\mathbf{\%})$ & All Patients $(\boldsymbol{n}=\mathbf{3 1})$ & Single Agent $(\boldsymbol{n}=\mathbf{1 6})$ & Combination $(\boldsymbol{n}=\mathbf{1 5})$ \\
\hline Progressive Disease & $15(48.4 \%)$ & $7(43.8 \%)$ & $8(53.3 \%)$ \\
\hline Stable Disease & $7(22.6 \%)$ & $4(25 \%)$ & $3(20 \%)$ \\
\hline Partial Response & $2(6.5 \%)$ & $1(6.3 \%)$ & $1(6.7 \%)$ \\
\hline Complete Response & 0 & 0 & 0 \\
\hline Non-evaluable & $7(22.6 \%)$ & $4(25 \%)$ & $6.7 \%$ \\
\hline $\begin{array}{c}\text { Objective Response } \\
\text { Rate }\end{array}$ & $6.5 \%$ & $6.3 \%$ & $26.7 \%$ \\
\hline Disease Control Rate & $29.0 \%$ & $31.3 \%$ &
\end{tabular}

\subsection{Exploratory Analysis of Patients on Cabozantinib-ICI Combinations}

In real-life practice, 15 patients $(35.7 \%)$ received cabozantinib in combination with ICIs. All of them had cabozantinib added to the ongoing ICI regime as add-on therapy after having progressive disease on the prior ICI regime. Add-on patients received prior ICIs for a longer time (the median duration on previous ICIs was 44.1 weeks (range 8-184.6), vs. 15.25 weeks (range $2-46$ ), $p=0.009$ ) compared to single-agent post-ICI patients. In this population, an overall ORR of $6.7 \%$ and DCR of $26.7 \%$ were observed (Table 4 ). The median TTP was 2.27 months (95\% C.I., 1.40-3.14) and median OS was 15.1 months (95\% C.I., 11.1-19.2) (Figure 1). The 6-month and 1-year survival rates were 79.4\% and $71.5 \%$, respectively. A total of $73.3 \%$ of such patients had any grade AEs while $13.3 \%$ had grade $\geq 3$ AEs. The most common AE was HFS. Twenty percent of patients required a dose reduction and $13.3 \%$ required treatment cessation due to AEs (Table 3 ).

\section{Discussion}

In our present study, we reported an ORR of $3.7 \%$, DCR of $44.4 \%$, median TTP of 3.88 months, and a median OS of 8.28 months in aHCC patients who received singleagent cabozantinib in a real-life setting. Importantly, our cohort provided salient data on the use of cabozantinib in a contemporary set of patients who progressed after various recently approved TKIs and ICIs. We also observed an interesting pattern of real-life use of cabozantinib as an add-on therapy to patients who progressed on ICIs.

The VEGF/VEGFR pathway is heavily implicated in HCC pathogenesis [2]. VEGFR-2 binds to VEGF-A to D, causing endothelial proliferation and angiogenesis and facilitating tumor progression [2]. Tumors with high VEGF levels have higher micro vessel density, more aggressive progression, and poorer survival [26-28]. Blockade of VEGF pathways can lead to up-regulation of the c-Met pathway. c-Met is a tyrosine kinase receptor for hepatocyte-growth factor (HGF) [29]. It is induced by hypoxia-inducible factor-1 (HIF-1) and involved in hepatic repair in chronic liver disease by inducing hepatocyte regeneration and suppressing chronic inflammation [30-33]. However, aberrant c-Met activation drives HCC pathogenesis and progression through promotion of cellular proliferation, invasion, and resistance to VEGF pathway blockade [9,34]. Cirrhosis increases c-MET activity through inducing HIF-1 by tissue hypoxia and increasing hepatocyte regeneration [29]. HCC further increases c-Met activity through increasing genomic instability and HGF levels [29,35]. In fact, c-Met aberrations are found in 50\% of all HCCs [29]. By primarily targeting VEGFR-2 and c-Met pathways, cabozantinib can block multiple oncogenic, angiogenic, and escape pathways and exert anti-tumor effects on HCCs [10].

In the CELESTIAL trial, an ORR of $4 \%$ and a median OS of 10.2 months were observed with single-agent cabozantinib [14]. In another single-arm phase II trial, Kudo et al. reported an ORR of $0 \%$, DCR of $76.5 \%$, and an OS rate at 6 months of $91.1 \%$ in 34 patients 
with aHCC who were mainly pretreated with sorafenib or lenvatinib, and with cabozantinib exclusively used in the second- or third-line setting [36]. Two other studies also reported the real-life use of single-agent cabozantinib [37,38]. In a cohort of 52 patients with CP-A liver function, mostly $(80.8 \%)$ aHCC, and with cabozantinib mostly in a third-line setting $(88.0 \%)$, Tovoli et al. reported a DCR of $59.2 \%$ and a median OS of 12.9 months. Meanwhile, Finkelmeier et al. reported a median OS of 7.7 months in a cohort of 74 patients with cabozantinib mainly as second- (50\%) and third-line (34\%) treatment, with $5 \%$ and $30 \%$ of patients achieving partial response and stable disease, respectively. Generally speaking, the results from our analysis were quite comparable with similar response rate and survival. Furthermore, it is worthwhile to note that single-agent cabozantinib was, in general, well tolerated in our study, with grade 3-4 AEs occurring in only $7.4 \%$ of such patients. The profile of AEs was similar to other published studies of cabozantinib. Interestingly, the rates of grade 3-4 AEs and AEs requiring dose reduction were all lower in our study than in other published trials $[14,36]$. This may reflect the differences in collection of AEs in real-life practice compared to clinical trial settings. An alternative explanation is that this may be due to a difference in dosing strategies in a real-life setting compared to in clinical trials, as cabozantinib was generally started at lower dose and titrated up to $60 \mathrm{mg}$ daily if tolerated in our patients, whilst treatment was generally commenced at $60 \mathrm{mg}$ directly in trials with subsequent dose reduction if needed. In fact, the majority of our patients only received an average daily dose of $40 \mathrm{mg}$ or below.

There is an urgent need to establish effective therapy for patients who are refractory to ICI based treatments. In the CELESTIAL trial, 14 patients who had received prior sorafenib and anti-PD-1/L1 were put on cabozantinib. In this subgroup, a median OS of 7.9 months and $64 \%$ of patients having grade 3 or 4 AEs were reported [22]. Post-ICI TKIs were also recently described in patients who received sorafenib, lenvatinib, or cabozantinib as second-line treatment after first-line atezolizumab-bevacizumab, demonstrating an ORR of $6.1 \%$, DCR of $63.3 \%$, and a median OS of 14.7 months. However, only one patient with cabozantinib was included in this study [39]. To our knowledge, no other literature exists in regard to post-ICI use of cabozantinib. However, several phase II trials for single-agent cabozantinib in this setting are currently underway $[40,41]$. In our study, we demonstrated that post-ICI use of single-agent cabozantinib can achieve good tumor control, survival, and tolerability. Moreover, in real-life practice, cabozantinib was also used as add-on therapy to ICIs. Notably, those who received cabozantinib as add-on therapy had been treated with prior ICI for a longer duration. This may be due to clinicians seeing benefits in continuing ICIs beyond progression due to good tolerability and indolent disease progression. Therefore, they were more inclined to add cabozantinib to ICIs rather than switching to other new therapies even after progression. In fact, there is an increasing amount of evidence of synergism between cabozantinib and ICIs in the literature. It was recently shown that c-Met inhibition by tivantinib/capmatinib reduces GSK3Bmediated PDL-1 degradation, resulting in increased PDL-1 expression and inactivation of co-cultured T cells in the HCC cell line, as well as reduced anti-tumor activity of T cells in mouse models [42]. Compared to anti-PD1 or c-Met inhibitors alone, combined treatment of c-Met inhibitors with anti-PD-1 increased tumor-infiltrating CD8+ T cells, decreased tumor growth, and prolonged survival of such mice [42]. Moreover, early clinical trial results demonstrated synergistic action of cabozantinib plus ICIs in aHCC. In the results of the CheckMate-040 trial cohort 6, nivolumab-cabozantinib and nivolumab-ipilimumabcabozantinib demonstrated ORRs of $17 \%$ and $26 \%$, DCRs of $81 \%$ and $83 \%$, as well as a median PFS of 5.5 and 6.8 months, respectively [43]. The median OS was 21.5 months for the doublet arm and not reached for the triplet arm, respectively. Additionally, the COSMIC-312 trial, which assessed the efficacy and safety of atezolizumab/cabozantinib as first-line treatment in aHCC patients, completed its recruitment [44]. The mature results from COSMIC-312 will provide the HCC community with important information about potential synergistic activities of cabozantinib with ICIs. Although the involved number of patients were small, the efficacy, survival, and tolerability achieved by this approach 
was encouraging. Nevertheless, it should be noted that the practice and benefits of using add-on cabozantinib to ICIs is not supported by any available phase III clinical trial data and was only an interesting phenomenon that we observed in this real-life study.

This study has several limitations, including small sample size, heterogenous population, biases inherent in its retrospective nature, and lack of blinded, independent review of treatment responses. The subgroup analyses contained a small number of patients, and large-scale studies are needed to validate their findings. Owing to limitations inherent to real-life practice, reassessment imaging was only planned every 8-12 weeks instead of every 6 weeks per available data. Additionally, the majority of our patients only received $20-40 \mathrm{mg} /$ day of cabozantinib instead of the registration dose of $60 \mathrm{mg} /$ day due to safety concerns in a real-life setting. Furthermore, the usage of cabozantinib as a single agent beyond the third line, or with ICIs beyond the first line, is not evidence-based and needs further clarification. The cabozantinib combinations used were heterogenous and have not been validated beyond the preclinical stage. These factors limit the study to hypothesisgenerating only and precludes direct recommendation of the use of cabozantinib in aHCC beyond its current licensed indication.

\section{Conclusions}

In conclusion, this study demonstrated that real-life use of cabozantinib can achieve encouraging anti-tumor activity and survival outcomes with good tolerability in aHCC patients in second line or beyond. Importantly, we also showed that post-ICI use of cabozantinib either as a single agent or as add-on therapy to ICIs can achieve tumor control, good safety profiles, and potential survival benefits. The results of ongoing prospective studies will verify our findings and address the unmet medical needs.

Supplementary Materials: The following are available online at https:/ / www.mdpi.com/article/ 10.3390/cancers13092002/s1, Table S1. Detailed prior systemic treatments. Table S2. Cabozantinib combination details.

Author Contributions: J.S.-L.W., Y.D., and T.Y. conceived and designed the study. J.S.-L.W., Y.D., V.T., T.L., C.S.Y.Y., A.T., A.L., T.S., G.G.-W.K., B.C.-W.L., R.L., J.C., K.-W.M., W.-H.S., J.T., T.-T.C. and T.Y. recruited patients and collected the data. J.S.-L.W., Y.D., V.T. and T.Y. analyzed the data. All authors have read and agreed to the published version of the manuscript.

Funding: This study was funded by the Research Grant Council-Theme Based Research Fund (T12-704/16-R) and Ipsen education grant.

Institutional Review Board Statement: This study was approved by The University of Hong Kong/Hospital Authority Hong Kong West Cluster Institutional Review Board (IRB Number UW 20-787).

Informed Consent Statement: This is a retrospective study without clinical intervention. Consent requirement has been deemed unnecessary by the relevant institutional review board.

Data Availability Statement: The data presented in this study are not publicly available for privacy and legal reasons.

Acknowledgments: We thank the patients and their families, investigators, and research staff at Queen Mary Hospital, Hong Kong. We thank the Department of Medicine, Queen Mary Hospital, and the University of Hong Kong for funding support.

Conflicts of Interest: T. Y. reports receiving honoraria from Ipsen and Exelixis. T.Y. has a consulting or advisory role at Ipsen and Exelixis. J.S.L.W., Y.D., V.T., T.L., C.S.Y.Y., A.T., A.L., T.S., G.G.W.K., B.C.W.L., R.L., J.C., K.W.M., W.H.S., J.T. and T.T.C have nothing to disclose.

\section{References}

1. She, W.H.; Chok, K. Strategies to increase the resectability of hepatocellular carcinoma. World J. Hepatol. 2015, 7, $2147-2154$. [CrossRef]

2. Morse, M.A.; Sun, W.; Kim, R.; He, A.R.; Abada, P.B.; Mynderse, M.; Finn, R.S. The Role of Angiogenesis in Hepatocellular Carcinoma. Clin. Cancer Res. 2019, 25, 912-920. [CrossRef] 
3. Llovet, J.M.; Ricci, S.; Mazzaferro, V.; Hilgard, P.; Gane, E.; Blanc, J.F.; de Oliveira, A.C.; Santoro, A.; Raoul, J.L.; Forner, A.; et al. Sorafenib in advanced hepatocellular carcinoma. N. Engl. J. Med. 2008, 359, 378-390. [CrossRef]

4. Kudo, M.; Finn, R.S.; Qin, S.; Han, K.H.; Ikeda, K.; Piscaglia, F.; Baron, A.; Park, J.W.; Han, G.; Jassem, J.; et al. Lenvatinib versus sorafenib in first-line treatment of patients with unresectable hepatocellular carcinoma: A randomised phase 3 non-inferiority trial. Lancet 2018, 391, 1163-1173. [CrossRef]

5. Bruix, J.; Qin, S.; Merle, P.; Granito, A.; Huang, Y.H.; Bodoky, G.; Pracht, M.; Yokosuka, O.; Rosmorduc, O.; Breder, V.; et al. Regorafenib for patients with hepatocellular carcinoma who progressed on sorafenib treatment (RESORCE): A randomised, double-blind, placebo-controlled, phase 3 trial. Lancet 2017, 389, 56-66. [CrossRef]

6. Zhu, A.X.; Kang, Y.K.; Yen, C.J.; Finn, R.S.; Galle, P.R.; Llovet, J.M.; Assenat, E.; Brandi, G.; Pracht, M.; Lim, H.Y.; et al. Ramucirumab after sorafenib in patients with advanced hepatocellular carcinoma and increased alpha-fetoprotein concentrations (REACH-2): A randomised, double-blind, placebo-controlled, phase 3 trial. Lancet Oncol. 2019, 20, 282-296. [CrossRef]

7. Rankin, E.B.; Giaccia, A.J. The Receptor Tyrosine Kinase AXL in Cancer Progression. Cancers 2016, 8, 103. [CrossRef]

8. Gherardi, E.; Birchmeier, W.; Birchmeier, C.; Vande Woude, G. Targeting MET in cancer: Rationale and progress. Nat. Rev. Cancer 2012, 12, 89-103. [CrossRef] [PubMed]

9. Boccaccio, C.; Comoglio, P.M. Invasive growth: A MET-driven genetic programme for cancer and stem cells. Nat. Rev. Cancer 2006, 6, 637-645. [CrossRef] [PubMed]

10. Xiang, Q.; Chen, W.; Ren, M.; Wang, J.; Zhang, H.; Deng, D.Y.; Zhang, L.; Shang, C.; Chen, Y. Cabozantinib suppresses tumor growth and metastasis in hepatocellular carcinoma by a dual blockade of VEGFR2 and MET. Clin. Cancer Res. 2014, 20, 2959-2970. [CrossRef]

11. Rimassa, L.; Assenat, E.; Peck-Radosavljevic, M.; Pracht, M.; Zagonel, V.; Mathurin, P.; Rota Caremoli, E.; Porta, C.; Daniele, B.; Bolondi, L.; et al. Tivantinib for second-line treatment of MET-high, advanced hepatocellular carcinoma (METIV-HCC): A final analysis of a phase 3, randomised, placebo-controlled study. Lancet Oncol. 2018, 19, 682-693. [CrossRef]

12. Firtina Karagonlar, Z.; Koc, D.; Iscan, E.; Erdal, E.; Atabey, N. Elevated hepatocyte growth factor expression as an autocrine c-Met activation mechanism in acquired resistance to sorafenib in hepatocellular carcinoma cells. Cancer Sci. 2016, 107, 407-416. [CrossRef]

13. Yakes, F.M.; Chen, J.; Tan, J.; Yamaguchi, K.; Shi, Y.; Yu, P.; Qian, F.; Chu, F.; Bentzien, F.; Cancilla, B.; et al. Cabozantinib (XL184), a novel MET and VEGFR2 inhibitor, simultaneously suppresses metastasis, angiogenesis, and tumor growth. Mol. Cancer Ther. 2011, 10, 2298-2308. [CrossRef] [PubMed]

14. Abou-Alfa, G.K.; Meyer, T.; Cheng, A.L.; El-Khoueiry, A.B.; Rimassa, L.; Ryoo, B.Y.; Cicin, I.; Merle, P.; Chen, Y.; Park, J.W.; et al. Cabozantinib in Patients with Advanced and Progressing Hepatocellular Carcinoma. N. Engl. J. Med. 2018, 379, 54-63. [CrossRef] [PubMed]

15. Finn, R.S.; Qin, S.; Ikeda, M.; Galle, P.R.; Ducreux, M.; Kim, T.-Y.; Lim, H.Y.; Kudo, M.; Breder, V.V.; Merle, P.; et al. IMbrave150: Updated overall survival (OS) data from a global, randomized, open-label phase III study of atezolizumab (atezo) + bevacizumab (bev) versus sorafenib (sor) in patients (pts) with unresectable hepatocellular carcinoma (HCC). J. Clin. Oncol. 2021, 39 (Suppl. 3), 267. [CrossRef]

16. Finn, R.S.; Qin, S.; Ikeda, M.; Galle, P.R.; Ducreux, M.; Kim, T.Y.; Kudo, M.; Breder, V.; Merle, P.; Kaseb, A.O.; et al. Atezolizumab plus Bevacizumab in Unresectable Hepatocellular Carcinoma. N. Engl. J. Med. 2020, 382, 1894-1905. [CrossRef]

17. El-Khoueiry, A.B.; Sangro, B.; Yau, T.; Crocenzi, T.S.; Kudo, M.; Hsu, C.; Kim, T.Y.; Choo, S.P.; Trojan, J.; Welling, T.H.R.; et al. Nivolumab in patients with advanced hepatocellular carcinoma (CheckMate 040): An open-label, non-comparative, phase $1 / 2$ dose escalation and expansion trial. Lancet 2017, 389, 2492-2502. [CrossRef]

18. Yau, T.; Kang, Y.K.; Kim, T.Y.; El-Khoueiry, A.B.; Santoro, A.; Sangro, B.; Melero, I.; Kudo, M.; Hou, M.M.; Matilla, A.; et al. Efficacy and Safety of Nivolumab Plus Ipilimumab in Patients with Advanced Hepatocellular Carcinoma Previously Treated With Sorafenib: The CheckMate 040 Randomized Clinical Trial. JAMA Oncol. 2020, 6, e204564. [CrossRef] [PubMed]

19. Zhu, A.X.; Finn, R.S.; Edeline, J.; Cattan, S.; Ogasawara, S.; Palmer, D.; Verslype, C.; Zagonel, V.; Fartoux, L.; Vogel, A.; et al. Pembrolizumab in patients with advanced hepatocellular carcinoma previously treated with sorafenib (KEYNOTE-224): A non-randomised, open-label phase 2 trial. Lancet Oncol. 2018, 19, 940-952. [CrossRef]

20. Finn, R.S.; Ryoo, B.Y.; Merle, P.; Kudo, M.; Bouattour, M.; Lim, H.Y.; Breder, V.; Edeline, J.; Chao, Y.; Ogasawara, S.; et al. Pembrolizumab As Second-Line Therapy in Patients with Advanced Hepatocellular Carcinoma in KEYNOTE-240: A Randomized, Double-Blind, Phase III Trial. J. Clin. Oncol. 2020, 38, 193-202. [CrossRef] [PubMed]

21. Yau, T.; Park, J.W.; Finn, R.S.; Cheng, A.L.; Mathurin, P.; Edeline, J.; Kudo, M.; Han, K.H.; Harding, J.J.; Merle, P.; et al. CheckMate 459: A randomized, multi-center phase III study of nivolumab (NIVO) vs sorafenib (SOR) as first-line (1L) treatment in patients (pts) with advanced hepatocellular carcinoma (aHCC). Ann. Oncol. 2019, 30, v874-v875. [CrossRef]

22. Abou-Alfa, G.K.; Cheng, A.L.; Saletan, S.; Kelley, R.K.; El-Khoueiry, A.B. Cabozantinib in Patients with Advanced Hepatocellular Carcinoma Previously Treated with anti-VEGF and Immuno-Oncology Therapy: Subgroup Analysis from the Phase 3 CELESTIAL Trial; Liver Cancer Summit, European Association for the Study of the Liver: Prague, Czech Republic, 2020.

23. Eisenhauer, E.A.; Therasse, P.; Bogaerts, J.; Schwartz, L.H.; Sargent, D.; Ford, R.; Dancey, J.; Arbuck, S.; Gwyther, S.; Mooney, M.; et al. New response evaluation criteria in solid tumours: Revised RECIST guideline (version 1.1). Eur. J. Cancer 2009, 45, $228-247$. [CrossRef] 
24. Lencioni, R.; Llovet, J.M. Modified RECIST (mRECIST) assessment for hepatocellular carcinoma. Semin. Liver Dis. 2010, 30, 52-60. [CrossRef]

25. National Cancer Institute (U.S.). Common Terminology Criteria for Adverse Events (CTCAE), Rev. ed.; U.S. Dept. of Health and Human Services, National Institutes of Health, National Cancer Institute: Bethesda, MD, USA, 2009; p. 194.

26. Poon, R.T.; Fan, S.T.; Wong, J. Clinical implications of circulating angiogenic factors in cancer patients. J. Clin. Oncol 2001, 19, 1207-1225. [CrossRef] [PubMed]

27. Poon, R.T.; Fan, S.T.; Wong, J. Clinical significance of angiogenesis in gastrointestinal cancers: A target for novel prognostic and therapeutic approaches. Ann. Surg. 2003, 238, 9-28. [CrossRef] [PubMed]

28. Amini, A.; Masoumi Moghaddam, S.; Morris, D.L.; Pourgholami, M.H. The critical role of vascular endothelial growth factor in tumor angiogenesis. Curr. Cancer Drug Targets 2012, 12, 23-43. [CrossRef] [PubMed]

29. Bouattour, M.; Raymond, E.; Qin, S.; Cheng, A.L.; Stammberger, U.; Locatelli, G.; Faivre, S. Recent developments of c-Met as a therapeutic target in hepatocellular carcinoma. Hepatology 2018, 67, 1132-1149. [CrossRef]

30. Noguchi, O.; Enomoto, N.; Ikeda, T.; Kobayashi, F.; Marumo, F.; Sato, C. Gene expressions of c-met and hepatocyte growth factor in chronic liver disease and hepatocellular carcinoma. J. Hepatol. 1996, 24, 286-292. [CrossRef]

31. Marquardt, J.U.; Seo, D.; Gomez-Quiroz, L.E.; Uchida, K.; Gillen, M.C.; Kitade, M.; Kaposi-Novak, P.; Conner, E.A.; Factor, V.M.; Thorgeirsson, S.S. Loss of c-Met accelerates development of liver fibrosis in response to $\mathrm{CCl}(4)$ exposure through deregulation of multiple molecular pathways. Biochim. Biophys. Acta 2012, 1822, 942-951. [CrossRef]

32. Borowiak, M.; Garratt, A.N.; Wustefeld, T.; Strehle, M.; Trautwein, C.; Birchmeier, C. Met provides essential signals for liver regeneration. Proc. Natl. Acad. Sci. USA 2004, 101, 10608-10613. [CrossRef]

33. Okano, J.; Shiota, G.; Kawasaki, H. Expression of hepatocyte growth factor (HGF) and HGF receptor (c-met) proteins in liver diseases: An immunohistochemical study. Liver 1999, 19, 151-159. [CrossRef]

34. Lee, S.J.; Lee, J.; Sohn, I.; Mao, M.; Kai, W.; Park, C.K.; Lim, H.Y. A survey of c-MET expression and amplification in 287 patients with hepatocellular carcinoma. Anticancer Res. 2013, 33, 5179-5186.

35. Vejchapipat, P.; Tangkijvanich, P.; Theamboonlers, A.; Chongsrisawat, V.; Chittmittrapap, S.; Poovorawan, Y. Association between serum hepatocyte growth factor and survival in untreated hepatocellular carcinoma. J. Gastroenterol 2004, 39, 1182-1188. [CrossRef]

36. Kudo, M.; Tsuchiya, K.; Kato, N.; Hagihara, A.; Numata, K.; Aikata, H.; Inaba, Y.; Kondo, S.; Motomura, K.; Furuse, J.; et al. Cabozantinib in Japanese patients with advanced hepatocellular carcinoma: A phase 2 multicenter study. J. Gastroenterol. 2021, 56, 181-190. [CrossRef] [PubMed]

37. Tovoli, F.; Dadduzio, V.; De Lorenzo, S.; Garajovà, I.; Marra, F.; Trevisani, F.; Pini, S.; Granito, A.; Zagonel, V.; Brandi, G.; et al. 999P Real-life clinical data of cabozantinib for unresectable hepatocellular carcinoma. Ann. Oncol. 2020, 31, S695. [CrossRef]

38. Finkelmeier, F.; Scheiner, B.; Leyh, C.; Best, J.; Fründt, T.W.; Czauderna, C.; Beutel, A.; Bettinger, D.; Weiß, J.; Meischl, T.; et al. Cabozantinib in advanced hepatocellular carcinoma: Efficacy and safety data from an international multicenter real-world cohort. J. Clin. Oncol. 2020, 38 (Suppl. 15), e16668. [CrossRef]

39. Yoo, C.; Kim, J.H.; Ryu, M.-H.; Park, S.R.; Lee, D.; Kim, K.M.; Shim, J.H.; Lim, Y.-S.; Lee, H.C.; Lee, J.; et al. Clinical Outcomes with Multikinase Inhibitors after Progression on First-Line Atezolizumab Plus Bevacizumab in Patients with Advanced Hepatocellular Carcinoma: A Multinational, Multicenter Retrospective Study. Liver Cancer 2021, 10, 107-114. [CrossRef]

40. Efficacy and Safety of Cabozantinib in Patients With Hepatocellular Carcinoma. ClinicalTrials.gov identifier NCT04435977. Available online: https:/ / clinicaltrials.gov / ct2/show / NCT04435977 (accessed on 23 March 2021).

41. Cabozantinib in Patients With Hepatocellular Carcinoma (ACTION). ClinicalTrails.gov identifier NCT04316182. Available online: https: / / clinicaltrials.gov/ct2/show / NCT04316182 (accessed on 23 March 2021).

42. Li, H.; Li, C.W.; Li, X.; Ding, Q.; Guo, L.; Liu, S.; Liu, C.; Lai, C.C.; Hsu, J.M.; Dong, Q.; et al. MET Inhibitors Promote Liver Tumor Evasion of the Immune Response by Stabilizing PDL1. Gastroenterology 2019, 156, 1849-1861.e13. [CrossRef]

43. Yau, T.; Zagonel, V.; Santoro, A.; Acosta-Rivera, M.; Choo, S.P.; Matilla, A.; He, A.R.; Gracián, A.C.; El-Khoueiry, A.B.; Sangro, B.; et al. Nivolumab (NIVO) + ipilimumab (IPI) + cabozantinib (CABO) combination therapy in patients (pts) with advanced hepatocellular carcinoma (aHCC): Results from CheckMate 040. J. Clin. Oncol. 2020, 38 (Suppl. 4), 478. [CrossRef]

44. Kelley, R.K.; Oliver, J.W.; Hazra, S.; Benzaghou, F.; Yau, T.; Cheng, A.L.; Rimassa, L. Cabozantinib in combination with atezolizumab versus sorafenib in treatment-naive advanced hepatocellular carcinoma: COSMIC-312 Phase III study design. Future Oncol. 2020, 16, 1525-1536. [CrossRef] 